\title{
Surface Acoustic Wave Vibration Sensors for Linear Electronic Warning Systems
}

\author{
J. Filipiak ${ }^{a, *}$, L. Solarz ${ }^{b}$ And G. SteczKo ${ }^{a}$ \\ ${ }^{a}$ Institute of Electronic and Control Systems, Technical University of Częstochowa \\ Armii Krajowej 17, 02-240 Częstochowa, Poland \\ ${ }^{b}$ Department of Civil Engineering, Military University of Technology, Kaliskiego 2, 00-908 Warsaw, Poland
}

The topic of the paper is analysis of feasibility of surface acoustic wave vibration sensors for linear electronic warning systems. In linear warning systems localisation of the object is realised by pointing out the sensors which detect vibrations caused by the object. The information sent out by the detector enables identification of the sensor and its state. The sensor contains surface acoustic wave delay line in four-terminal network. The delay line is formed at the surface of piezoelectric plate. The vibrations of the plate are caused by vibrations of the medium surrounding the sensor. The frequency of the input signal is equal to the working frequency of delay line. The phase of the output signal is shifted in comparison with the input signal. The frequency of the phase shift would be equal to the resonance frequency of the sensor plate. The change of the output signal amplitude would be proportional to the amplitude of plate vibrations. The measured amplitude and frequency of the output signal would be registered by simple electronic devices. The measurements give us knowledge which sensor vibrates and the intensity of these vibrations. Such sensors enable construction of the electronic warning system.

PACS numbers: 43.25.Fe, 77.65.Dq, 43.35.Pt, 68.35.Iv

\section{Introduction}

The main part of successful guarding is detection of people or vehicles approaching to a guarded area, such as airports, governmental buildings, fuel or dangerous fluids storage depots. The detection is realised by systems of sensors which may act when:

- an intruder stops a narrow stream of electromagnetic radiation, light, or other radiation

- an intruder generates vibrations in close surrounding of sensors.

The sensors systems of second kind are called linear. Among linear sensors we may enumerate: concentric sensor cables, fibre-optic cables, vibration sensors. Linear sensors signal when they vibrate or when the pressure acting at them changes. The surface acoustic wave vibration sensors (SAW-VSs) may work in such systems. Signals generated by SAW-VSs should contain information which of them is activated and how intensive its vibrations are.

In the paper, the sensors fulfilling the above mentioned requirements are proposed.

\section{Surface acoustic wave vibration sensor}

Sensors with SAW are used for measurements of many mechanical and physical values [1-10]. The most often

\footnotetext{
* corresponding author; e-mail: gem@gemsc.com.pl
}

SAW acceleration sensors are used [8-11]. The SAW acceleration sensors may be used to detect vibrations of the environment. The idea of the SAW acceleration sensor used as vibration sensor is explained in Fig. 1.

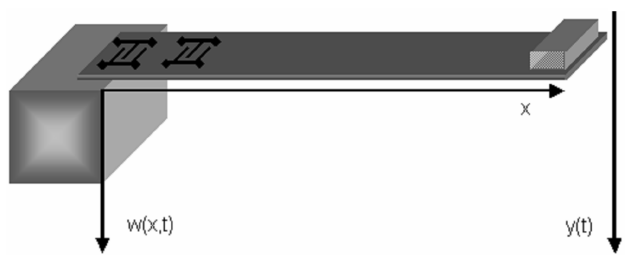

Fig. 1. The main elements of SAW-VS.

The plate made of an anisotropic piezoelectric medium is clamped to the casing. At the opposite end of the plate mass is attached. Two co-operating interdigital transducers are deposited on the surface of plate. One of them generates the surface acoustic wave. The second of them changes the mechanical disturbance into electrical signals. The whole system works as four-terminal network.

- Displacements caused by vibrations (bending) change the distance between the interdigital transducers and stress in the plate.

- Varying distance and stress change the time of delay of SAW delay line.

- Varying delay time changes phase shift of the electric signal in delay line. Frequency of the phase shift is equal to the resonance frequency of plate. 
Amplitude of the phase shift is proportional to the amplitude of plate vibrations.

Measuring the phase shift, we may measure resonance frequency of the plate, so we may know which sensor sends the signal, if they are not the same. Measuring the phase shift, we may measure intensity of vibrations caused by an intruder.

We consider the vibrations of the piezoelectric plate. To avoid difficult analysis of anisotropic plate we use the equivalent isotropic model described and analyzed in $[12,13]$. Elastic properties of the plate are described by equivalent Young modulus $E_{\mathrm{e}}$. Dissipation is quantitatively characterized by viscous model with parameter $\tau$. We also use equivalent system with one degree of freedom [14]. The qualitative and quantitative differences between applied model with one degree of freedom and the model with many degrees of freedom are explained in the next section of our paper. Because differences between the above-mentioned models are the greatest for plate without a mass at the end, the plate without such mass is considered.

\section{Comparison between models with one and many degrees of freedom applied to SAW vibration sensors}

We consider equivalent isotropic model of cylindrically bent plate $[12,13]$. Equation of free vibrations has the form

$$
\rho \frac{\partial^{2} w(x, t)}{\partial t^{2}}+E_{\mathrm{e}} \frac{h^{2}}{12}\left(1+\tau \frac{\partial}{\partial t}\right) \frac{\partial^{4} w(x, t)}{\partial t^{4}}=0,
$$

where $\rho$ - mass density, $h$ - thickness of the plate, $L$ - length of the plate.

At the boundaries we have

$$
\begin{aligned}
& w(0, t)=0, \quad \frac{\partial w(0, t)}{\partial x}=0, \quad \frac{\partial^{2} w(L, t)}{\partial x^{2}}=0, \\
& \frac{\partial^{3} w(L, t)}{\partial x^{3}}=0 .
\end{aligned}
$$

We assume that there is no additional mass at the boundary $x=L$. form

The solution to the boundary problem (1), (2) has the

$$
\begin{aligned}
& w(x, t) \\
& \quad=\sum_{n=1}^{\infty}\left[W_{n}(x) A_{n} \exp \left(-0.5 \omega_{n}^{2} \tau t\right) \sin \left(\bar{\omega}_{n} t+\varphi_{n}\right)\right] .
\end{aligned}
$$

The orthonormal set of function $W_{n}$ (eigenfunctions) is taken from [11]:

$$
\begin{aligned}
& W_{n}(x)=F_{n}\left[U\left(k_{n} \frac{x}{L}\right)-\frac{S\left(k_{n}\right)}{T\left(k_{n}\right)} V\left(k_{n} \frac{x}{L}\right)\right], \\
& F_{n}=\left[\int_{0}^{1}\left[U\left(k_{n} z\right)-\frac{S\left(k_{n}\right)}{T\left(k_{n}\right)} V\left(k_{n} z\right)\right]^{2} \mathrm{~d} z\right]^{-0.5},
\end{aligned}
$$

where the Krylov functions are

$$
S(\alpha)=\frac{1}{2}(\cos h \alpha+\cos \alpha),
$$

$$
\begin{aligned}
T(\alpha) & =\frac{1}{2}(\sin h \alpha+\sin \alpha), \\
U(\alpha) & =\frac{1}{2}(\cos h \alpha-\cos \alpha), \\
V(\alpha) & =\frac{1}{2}(\sin h \alpha+\sin \alpha), \\
k_{1} & =1.875, \quad k_{2}=4.694, \quad k_{3}=7.855 \ldots
\end{aligned}
$$

The angular frequency of non-damped vibrations is equal to

$$
\omega_{n}=k_{n}^{2} \frac{h}{L^{2}} \sqrt{\frac{E_{\mathrm{e}}}{12 \rho}} .
$$

The angular frequency of damped vibrations is equal to

$$
\bar{\omega}_{n}=\omega_{n} \sqrt{1-\frac{\omega_{n}^{2} \tau^{2}}{4}} .
$$

Only some elements in the sum (3) represent vibrations. For $N<n$, where $N$ is the greatest natural number for which $\omega_{N}<\frac{2}{\tau}$, the element of sum repre-

\begin{tabular}{|c|c|c|c|c|}
\hline \multirow{2}{*}{$\begin{array}{l}h \\
{[\mathrm{~mm}}\end{array}$} & \multirow{2}{*}{$\begin{array}{r}L \\
\mathrm{n}]\end{array}$} & \multirow[t]{2}{*}{$r$} & \multicolumn{2}{|c|}{ Normal frequency of the plate $[\mathrm{Hz}]$} \\
\hline & & & Quartz $-\mathrm{SiO}_{2}$ & Lithium niobate $-\mathrm{LiNbO}_{3}$ \\
\hline \multirow{5}{*}{0.5} & \multirow{5}{*}{15} & 0.0 & 1800 & 2300 \\
\hline & & 1.0 & 780 & 1000 \\
\hline & & 2.0 & 580 & 740 \\
\hline & & 3.0 & 480 & 720 \\
\hline & & 4.0 & 430 & 550 \\
\hline \multirow{6}{*}{0.5} & \multirow{6}{*}{65} & 0.0 & 82 & 106 \\
\hline & & 1.0 & 36 & 47 \\
\hline & & 2.0 & 27 & 35 \\
\hline & & 3.0 & 22 & 29 \\
\hline & & 4.0 & 19 & 25 \\
\hline & & 5.0 & 17 & 22.5 \\
\hline
\end{tabular}
sents very strongly damped movement and there is no resonance with this frequency. Each of the harmonics $n=1,2,3, \ldots$ has part of energy. How great is the part depends on the system (normal vibrations, $\omega_{n}, W_{n}(x)$ ) and depends on activation. Measurement of building vibrations caused by trains of Warsaw Metro disclosed that over $60 \mathrm{~Hz}$ waves are strongly damped. In grounds, this damping effect would be even stronger. The frequencies of the typical plates made of fused quartz or lithium niobate are enumerated in Table I. They are of the order of hundred of $\mathrm{Hz}$.

TABLE I

Normal frequencies $\omega_{1}$.

We expect excitation of vibrations with frequencies less than or equal to the frequency of the first harmonic so we expect that the calculations which take into account only one degree of freedom (one harmonic) would be good enough.

\section{System with SAW vibration sensors}

The SAW-VS cooperates with other SAW-VSs in the linear electronic warning system. Only one SAW-VS is seen in Fig. 2. 


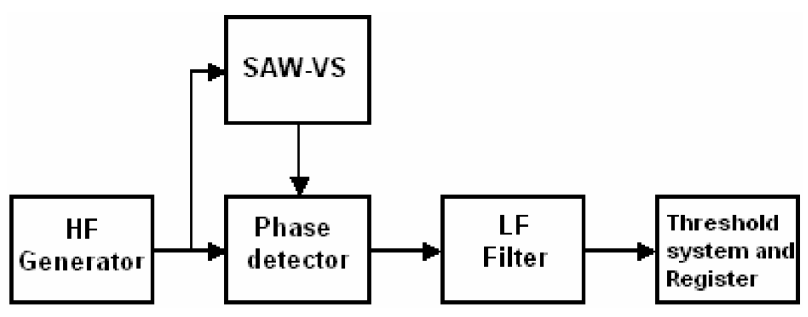

Fig. 2. The warning system with SAW-VS.

In the system seen in Fig. 2, the phase detector is fed with two signals:

- from the high frequency (HF) generator

$$
u_{\mathrm{g}}(t)=U_{\mathrm{g}} \sin \left(\omega_{\mathrm{d}} t\right),
$$

- from the SAW-VS

$$
u_{\mathrm{s}}(t)=U_{\mathrm{s}} \sin \left[\omega_{\mathrm{a}} t+\varphi_{\mathrm{s}}(t)\right],
$$

$\omega_{\mathrm{d}}=2 \pi f_{\mathrm{d}}$, where $f_{\mathrm{d}}$ is for delay-line frequency, $\varphi_{\mathrm{S}}$ is for phase shift.

At the output of phase detector we have:

- signals with the delay line frequency or even their harmonics,

- the signal with the resonance frequency of the SAW-VS,

- maybe the signals with the specific intruder frequency, too.

The abovementioned signals are at the input of low frequency (LF) filter. The characteristic of LF filter is matched to get the signal with resonance frequency of the piezoelectric plate only. This signal enables identification of the sensor. The amplitude of signal gives information about intensity of generated vibrations. The analysis of the amplitude and frequency domains of the signals is presented in the next chapter.

\section{The analysis of SAW-VS parameters}

Both SAW-VS intensity of vibrations and frequency depends on its dimensions, elasticity, and density and attenuation constants of the piezoelectric plate. The relations are derived at the base of model with one degree of freedom e.g. [15]. We know the impulse response

$$
A \exp \left(-0.5 \omega_{1}^{2} \tau t\right) \sin \left(\bar{\omega}_{1} t\right)
$$

where $\omega_{1}, \bar{\omega}_{1}$ are known from $(7),(8),(9)$ and we know amplitude-frequency characteristic (Fig. 3)

$$
H(\omega)=a\left\{\left[1-\left(\frac{\omega}{\omega_{1}}\right)^{2}\right]^{2}+(\omega \tau)^{2}\right\}^{-0.5},
$$

where $a$ is for static deflection.

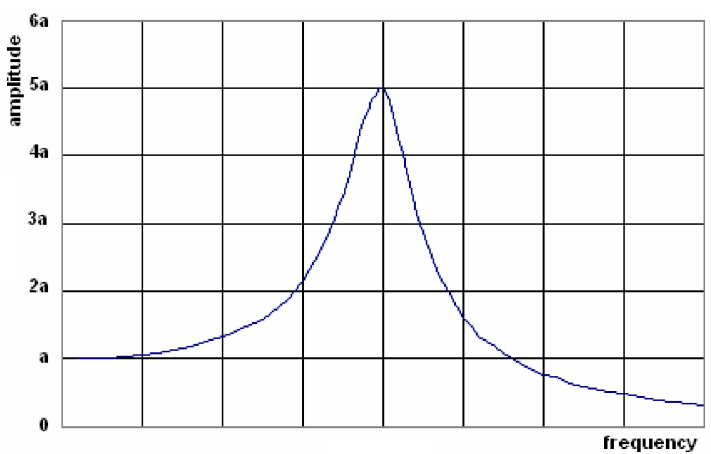

Fig. 3. The amplitude-frequency characteristic for a system with one degree of freedom.

The phase characteristic has the form (Fig. 4)

$$
\psi(\omega)=\operatorname{atan}\left\{(\omega \tau)\left[1-\left(\frac{\omega}{\omega_{1}}\right)^{2}\right]^{-1}\right\} .
$$

All the abovementioned characteristics depend on ratio $\omega / \omega_{1}$ and characteristic parameter $\tau$. The parameters needed in (8), (9) for $\omega_{1}$ are presented in Table II for different substrata.

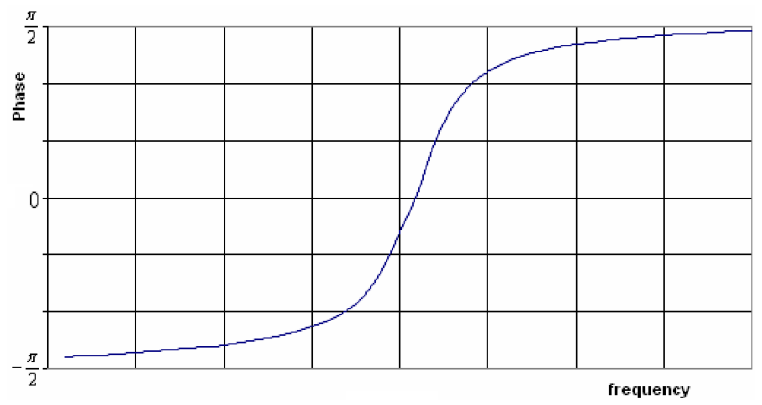

Fig. 4. The phase characteristic for a system with one degree of freedom.

TABLE II

Material constants. Quartz ST Cut, X prop.

\begin{tabular}{c|c|c|c}
\hline \hline & Quartz ST & $\begin{array}{c}\text { Lithium } \\
\text { niobate }\end{array}$ & $\begin{array}{c}\text { Lithium } \\
\text { tetraborate }\end{array}$ \\
\hline $\begin{array}{c}\text { equivalent Young } \\
\text { modulus } E_{\mathrm{e}}[\mathrm{GPa}]\end{array}$ & 76 & 215 & 120 \\
$\begin{array}{c}\text { critical stress [MPa] } \\
\text { characteristic damping } \\
\text { time } \tau[\mu \mathrm{s}]\end{array}$ & 80 & 55 & 60 \\
density $\left[\mathrm{kg} / \mathrm{m}^{3}\right]$ & 29.3 & 30 & 28.5 \\
\end{tabular}

Normal frequency $\omega_{1}$ calculated for different materials and dimensions are seen in Table I. Parameter $r$ is for the ratio of the mass at the end of plate to the mass of piezoelectric plate. The correction coefficient deduced by Rayleigh method in [9] is $(1+3.9689 r)^{-0.5}$. 
As is seen, the normal frequencies are in the range from $20 \mathrm{~Hz}$ to $2000 \mathrm{~Hz}$. The lowest frequencies are for great masses at the end of plate and the stresses are near the critical stress for the plate under dynamic loads.

The difference between normal frequency and resonance frequency is rather small. From the formula (9) and Tables I and III we may derive that the difference between 1 and the ratio $\bar{\omega}_{1} / \omega_{1}$ is in the range $\left(0.4 \times 10^{-6}-0.014\right)$. The data are for quartz ST. The effect is rather negligible for the first harmonic. As it was mentioned above, for higher harmonics, damping may exclude vibrations and resonance.

The time of vibration decay should be considered, too. The system has to register resonance frequency and the proper amount of time is needed for this purpose. The envelope of the signal is proportional to $\exp \left(-0.5 \omega_{1}^{2} \tau t\right)$. Let the symbol $T_{\mathrm{d}}$ be for the time when the signal diminishes e times. We assume that $\tau=25 \mu \mathrm{s}$. (Table II we have $28.5 \div 30 \mu \mathrm{s}$ ) and does not depend on frequency.

\section{TABLE III}

Decaying where $N$ is for number of periods in the time $T_{\mathrm{d}}$.

\begin{tabular}{c|c|c}
\hline \hline Frequency $[\mathrm{Hz}]$ & $T_{\mathrm{d}}[\mathrm{s}]$ & $N$ \\
\hline 20 & 5.066 & 101.3 \\
100 & 0.2026 & 20.26 \\
1000 & 0.002026 & 2.026
\end{tabular}

If the decay is too strong, there will exist problems with registration of frequency. More precise evaluation needs knowledge about electronic devices working in the system.

The stresses in plates should be lower than the critical stress for the chosen substratum (Table II). For harmonic vibrations the stresses are proportional to the square of frequency. So, especially for high frequency, we should be cautious and implement limiters for the amplitude of vibrations.

\section{Conclusions}

We analyse constructional solutions for surface acoustic wave vibration sensors working in linear electronic warning systems. The sensors should be activated, if disturbances are greater than experimentally specified threshold. The specific frequency of vibrations generated by the SAW-VS enables its identification in the system. This specific frequency depends on resonance frequency of the piezoelectric plate. The frequency should be in the domain $20 \div 400 \mathrm{~Hz}$. The lower limit of frequency results from the upper limit of crystal dimensions (see formulae (8) and (9)). The upper limit of frequency results from:

- decaying (Table III),

- the critical stress (Table II).
We may deposit SAW delay lines on the substrata enumerated in Table II as:

- two co-operating interdigital transducers,

- or resonator.

The electronic system coupled with them is rather different. An example SAW-VS with delay line working like resonator in four-terminal network is seen in Fig. 5 [5].

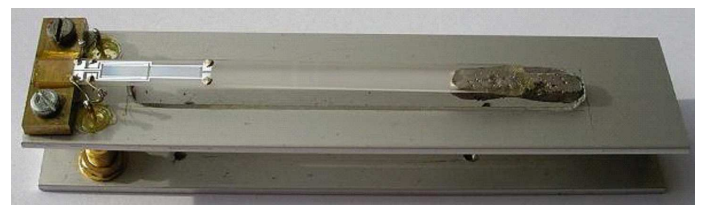

Fig. 5. SAW vibration sensor.

The working frequency of the resonator is $154 \mathrm{MHz}$. The delay line losses are $6.5 \mathrm{~dB}$. The resonance frequency of the plate is equal to $18 \mathrm{~Hz}$. The part of impulse response for the SAW-VS is seen in Fig. 6.

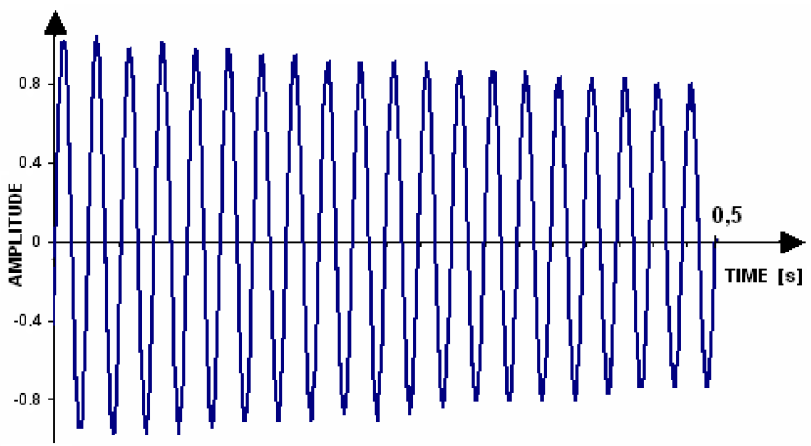

Fig. 6. The SAW-VS impulse response (part).

The losses for the delay line with two co-operating interdigital transducers would be far greater. In the case, the interdigital transducers should be matched to the coupled electronic devices. In both cases the delay line works as four-terminal network. The SAW-VS may be directly switched as cascade in linear warning electronic system.

\section{References}

[1] D. Hauden, S. Rousseau, J.J. Gagnepain, in: Proc. 34th IEEE Ann. Freq. Control Symph., NJ, 1988, p. 312 .

[2] T. Pustelny, B. Pustelny, Europ. Phys. J. - Special Topics 154, 265 (2008).

[3] E. Benes, M. Groshl, W. Burger, M. Schmid, Sensors Actuators A 45, 1 (1995).

[4] F. Seifert, W. Bulst, C. Ruppel, Sensors Actuators A 44, 231 (1994).

[5] W. Jakubik, M. Urbanczyk, E. Maciak, T. Pustelny, Bull. Pol. Acad. Sci. 56, 133 (2008). 
[6] D. Hauden, Arch. Acoust. 16, 17 (1991).

[7] T. Pustelny, A. Opilski, B. Pustelny, Acta Phys. Pol. A 114, A-183 (2008).

[8] J. Filipiak, SAW Acceleration Sensors, Czestochowa University of Technology Press, Częstochowa 2006 (in Polish).

[9] J. Filipiak, C. Kopycki, Sensors Actuators 76, 318 (1999).

[10] J. Filipiak, L. Solarz, G. Steczko, Mol. Quant. Acoust. 28, 71 (2007).
[11] J. Filipiak, L. Solarz, G. Steczko, Mol. Quant. Acoust. 28, 81 (2007).

[12] J. Filipiak, K. Zubko, Mol. Quant. Acoust. 26, 75 (2005).

[13] J. Filipiak, L. Solarz, K. Zubko, Mol. Quant. Acoust. 27, 79 (2006)

[14] J. Filipiak, L. Solarz, K. Zubko, Mol. Quant. Acoust. 25, 89 (2004).

[15] W. Bogusz, Z. Dżygadło, D. Rogula, K. Sobczyk, L. Solarz, S. Kaliski, in: Vibrations and Waves, Eds. S. Kaliski, L. Solarz, PWN-Elsevier, New York 1992. 